

University of Central Florida

STARS

Faculty Bibliography 2000s

Faculty Bibliography

$1-1-2002$

\title{
New phasing algorithm for large segmented telescope mirrors
}

Weiyao Zou

University of Central Florida

Find similar works at: https://stars.library.ucf.edu/facultybib2000

University of Central Florida Libraries http://library.ucf.edu

This Article is brought to you for free and open access by the Faculty Bibliography at STARS. It has been accepted for inclusion in Faculty Bibliography 2000 s by an authorized administrator of STARS. For more information, please contactSTARS@ucf.edu.

\section{Recommended Citation}

Zou, Weiyao, "New phasing algorithm for large segmented telescope mirrors" (2002). Faculty Bibliography 2000s. 3576.

https://stars.library.ucf.edu/facultybib2000/3576

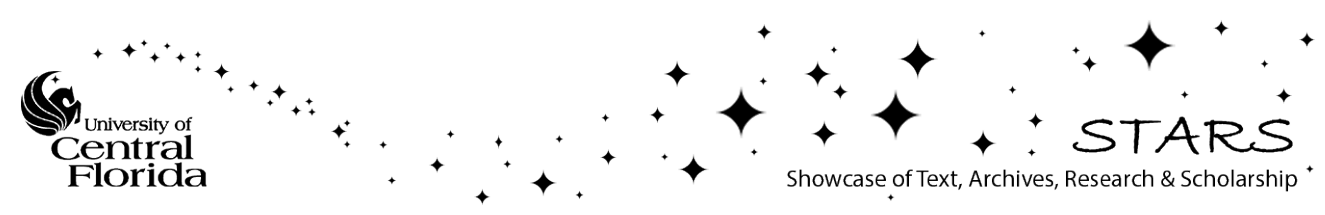




\section{New phasing algorithm for large segmented telescope mirrors}

\author{
Weiyao Zou \\ University of Central Florida \\ School of Optics/CREOL \\ P. O. Box 162700 \\ Orlando, Florida 32816-2700 \\ and \\ Nanjing Institute of Astronomical \\ Optics and Technology (NIAOT) \\ National Astronomical Observatories of \\ Chinese Academy of Sceiences (NAOC) \\ 188 Bancang Street \\ Ninjing, Jiangsu 210042 \\ China \\ E-mail:wzou@creol.ucf.edu
}

\begin{abstract}
We propose a new phase calibration method, called the sensor-by-sensor phase calibration method (SSPC), which is applied for the phasing of a large segmented telescope mirror. Instead of making the entire segmented mirror in phase at one time and obtaining the desired sensor readings accordingly, we serially make the local areas in phase at each sensor location and thus obtain the desired sensor readings one by one. Then we run the telescope active control system to make the segments cophase. For the case of Keck telescopes, the SSPC method uses all the 168 phase measurements at 168 phase sensor locations; then we obtain 168 desired sensor readings for the segmented mirror active control system. The SSPC method is completely insensitive to the residual tilt errors from the segment alignment and the residual errors from the active control system. The validity of the SSPC method has been demonstrated in an active optics experimental system, which has three segments and a 500-mm-diam overall aperture. The accuracy of the SSPC method is better than $20-\mathrm{mm}$ rms in this experimental system, and it is easy to obtain diffraction-limited images at a visible wavelength $\lambda=650 \mathrm{~nm}$ over an aperture of $220 \mathrm{~mm}$. (c) 2002 Society of Photo-Optical Instrumentation Engineers. [DOI: 10.1117/1.1497613]
\end{abstract}

Subject terms: phase calibration algorithm; large segmented telescope mirrors; active optics; figure control; telescopes.

Paper 010350 received Sep. 28, 2001; revised manuscript received Feb. 20, 2002; accepted for publication Feb. 20, 2002.

\section{Introduction}

Phase calibration is one of the most essential techniques in segmented mirror active optics. To achieve its full diffraction-limited performance, a segmented primary mirror must be properly phased. The segmented mirror employs a system of sensors and actuators to measure and correct the edge height differences between segments, which correspond to piston and tip/tilt errors. Because of its high resolution, a capacitive displacement sensor system is employed. For instance, the precision of the sensor used in Keck telescopes is $3 \mathrm{~nm} \mathrm{1sb}$, its measurement noise is 1-nm $\mathrm{rms}$, and its temporal drift is $3.2 \mathrm{~nm} /$ week. $^{1}$ They are installed under the segments to avoid blocking the lightcollection area.

However, the displacement sensors only indicate the relative changes between segment edges, which do not tell the phase status of the segments. Therefore, the desired sensor readings must be calibrated by other means. The goal of the phase calibration described is to determine the desired sensor readings at which the corresponding segment height differences are zero. Let us suppose the segment aberrations are small enough; if all the sensor readings are maintained at the desired sensor readings, and if all the segments are well aligned, the whole segmented mirror will be in phase and thereby reach its diffraction-limited imaging.

This work was inspired by the work done for Keck telescopes. ${ }^{2-4}$ The mirror configuration and sensor-actuator system designs employed here are similar to that of Keck telescopes. For their updated results and data, please refer to their newly published papers.

\section{Sensor-by-Sensor Phase Calibration (SSPC)}

\subsection{Strategy of SSPC}

To make a phase calibration, it is not necessary to make all the primary segments well cophased together before obtaining the desired sensor readings. We calibrate the sensor readings one by one, then make all the mirror segments in phase by the telescope innate active control system. The positions of all the segments are usually out of phase and "randomly" distributed when the entire mirror segments are just roughly aligned. It is difficult to make them in phase together in one step without the aid of active control, in which we need to know the desired sensor readings. However, if we focus our attention on one pair of adjacent segments, it is easy to make them in phase on a local intersegment subaperture (two lobes on two segments), where a sensor is installed on the back surface. We thereby obtain all the phase sensor readings (i.e., the sensor readings at which the segments are in phase) serially by repeatedly performing the local intersegmental area phasing one after another. After that, we apply the phase sensor readings as the desired sensor readings to the figure control equations. Then we run the active control system to improve the figure of the segmented mirror in iterations to make all segments 


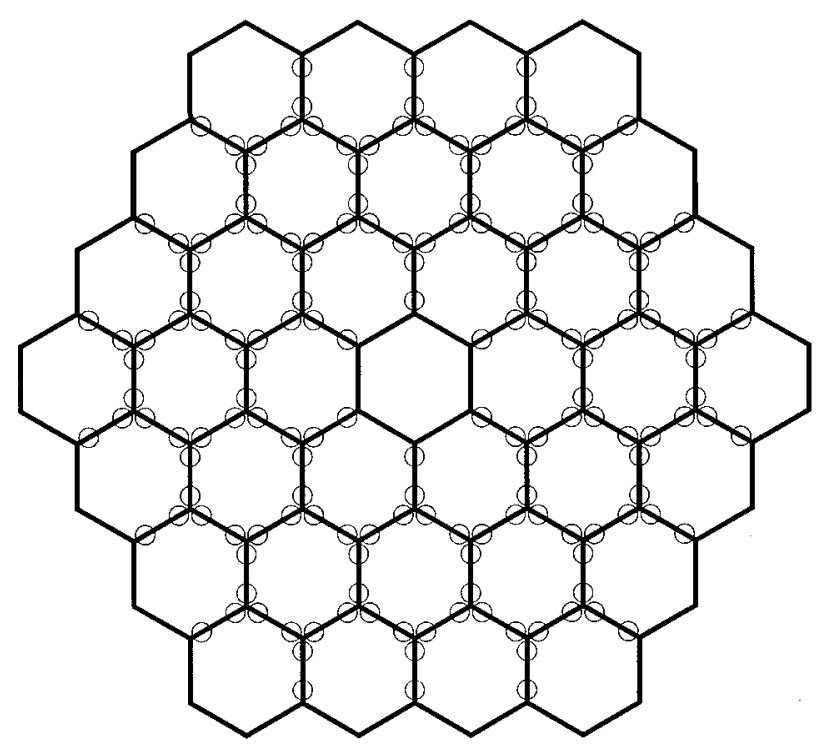

Fig. 1 The arrangement of calibration sites (168 subapertures total) of SSPC superposed on the image of a primary mirror with 36 segments.

in phase. We have chosen to call this serial phase calibration strategy the sensor-by-sensor phase calibration method, or SSPC.

Take a telescope mirror with 36 segments as an example: the distribution map of calibration locations would be shown as in Fig. 1. At first, the mirror figure is usually very "rough": the segments have both tilt and piston errors. Therefore, the precision of SSPC in the first iteration may not be very impressive. However, the active control system will dramatically improve the figure of the segmented mirror afterward, and we can get a much better precision of SSPC in the second iteration. Generally, a satisfactory result will be available after only two or three calibration-phasing iterations.

\subsection{Mathematics of Segmented Figure Control for SSPC}

As mentioned before in SSPC, we utilize the inherent equations of the active control system to correct the primary figure error; we do not need to separately built up another control system and phasing equations. Once the desired sensor readings are obtained, the process of phase calibration will be finished. How to control the actuators to phase the mirror segments is the task of the telescope's active control system. The figure control equations originally established for an active control system can be expressed as ${ }^{5,6}$

$\mathbf{A P}=\mathbf{S}-\mathbf{S}_{\text {in phase }}$

where $\mathbf{A}$ is the matrix of the figure control equations; $\mathbf{P}$ is the vector of the actuator correction steps; $\mathbf{S}$ is the vector of the real-time sensor readings; and $\mathbf{S}_{\text {in phase }}$ is the vector of the desired sensor readings obtained from the phase calibration. The displacement sensor system measures all the degrees of freedom of the segmented mirror except the degree of droop mode, which defines the overall droop distortion of the primary mirror geometry. The effect on the

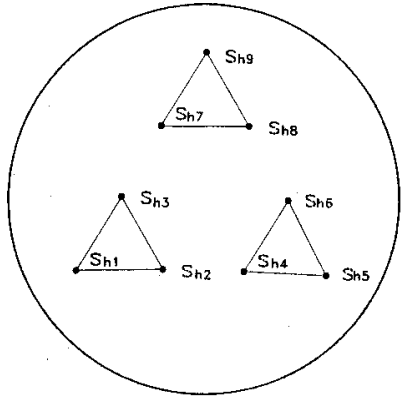

(a)

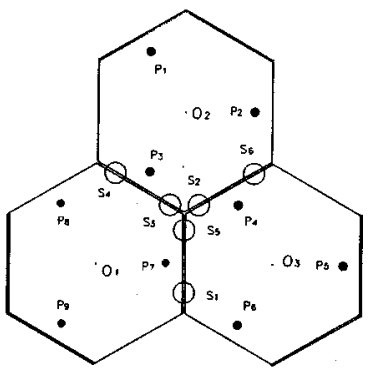

(b)

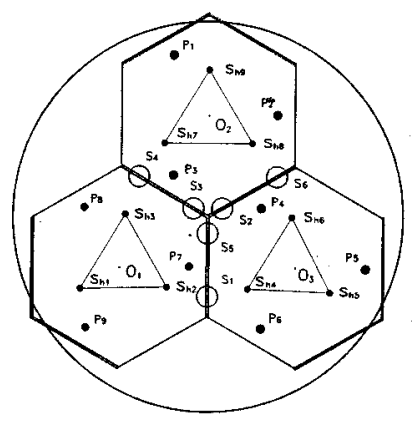

(c)

Fig. 2 Black dots $S h_{i}(i=1,2 \ldots 9)$ in (a) are Shack-Hartmann spots; and black dots $P_{i}(i=1,2, \ldots 9)$ in (b) are the actuator positions. The small intersegment circular apertures $S_{i}(i=1,2, \ldots 6)$ in (b) represent sensor positions on the back of the segments, and they are also the calibration sites for the circular-aperture method. The corresponding relationship from the Shack-Hartmann spot array to the mirror segments is evident if we superpose Figs. 2(a) and 2(b) in Fig. 2(c).

optical image of the droop mode distortion is simply a change of focus, at least for a small distortion. ${ }^{5}$

There are several algorithms available for phasing the mirror segments via the active control system, ${ }^{5-7}$ such as the minimum-norm least-squares method, the damped least-squares method, etc. Here we present a very fastconvergence algorithm for segment phasing for the SSPC method. The control equation for the mirror segment alignment can be expressed as

$\mathbf{A}_{F} \mathbf{P}=\mathbf{D}_{x y}$,

where $\mathbf{P}$ is a vector of actuator correction steps, $\mathbf{D}_{x y}$ is the vector of the coordinate differences between the measured spots and the standard spots of Shack-Hartmann on a CCD target, and $\mathbf{A}_{F}$ is the influence function of the segment alignment system. To improve the alignment precision, we employed a grid arrangement in the experimental system introduced in Sec. 3, in which each segment corresponds to the three Shack-Hartmann spots shown in Fig. 2. The influence function $\mathbf{A}_{F}$ varies as the $\mathrm{S}-\mathrm{H}$ grid scheme, the numbers of segments and actuators change. In fact, $\mathbf{A}_{F}$ is a matrix that is measured in experimentation. To phase the mirror segments, we should add come constraints to Eq. (1) for determining droop mode of the mirror. If we consider the figure control problem together with the segment alignment problem, we can define an evaluation function as

$\Psi(\mathbf{P})=\rho^{2}\left(\mathbf{A}_{F} \mathbf{P}, \mathbf{D}_{x y}\right)+\lambda \rho^{2}(\mathbf{A P}, \mathbf{S})$, 
where $\rho$ is defined as a distance (a norm) between two points (such as $x$ and $y$ ) in the Euclidean space, so

$\rho(x, y)=\left[\sum_{k=1}^{n}\left(x_{k}-y_{k}\right)^{2}\right]^{1 / 2}=\|x-y\|_{2}$.

Rewriting Eq. (3) in its norm form, we have

$\Psi(\mathbf{P})=\left\|\mathbf{A}_{F} \mathbf{P}-\mathbf{D}_{x y}\right\|_{2}^{2}+\lambda\|\mathbf{A P}-\mathbf{S}\|_{2}^{2}$,

where $\lambda>0$ is the weight factor between cophase and coalignment. From

$\frac{\mathrm{d} \Psi}{\mathrm{dP}}=0$,

we have

$$
\left(\mathbf{A}_{F}^{T} \mathbf{A}_{F}+\lambda \mathbf{A}^{T} \mathbf{A}\right) \mathbf{P}=\mathbf{A}_{F}^{T} \mathbf{D}_{x y}+\lambda \mathbf{A}^{T} \mathbf{S} .
$$

The value of $\lambda$ is determined in practice. To balance the two control processes, $\lambda$ is usually a big number; for the experimental system of Sec. 3, the value of $\lambda$ is about $10^{3} \sim 10^{4}$. Because of the independent relationship between Eqs. (1) and (2), the rank of the matrix $\mathbf{A}_{F}^{T} \mathbf{A}_{F}+\lambda \mathbf{A}^{T} \mathbf{A}$ is full, and its condition number is very good. Therefore, the least-squares solution of Eq. (7) can be expressed as

$$
\mathbf{P}=\left(\mathbf{A}_{F}^{T} \mathbf{A}_{F}+\lambda \mathbf{A}^{T} \mathbf{A}\right)^{-1}\left(\mathbf{A}_{F}^{T} \mathbf{D}_{x y}+\lambda \mathbf{A}^{T} \mathbf{S}\right) .
$$

This algorithm converges rapidly, and the precision of droop mode control here is higher than that of the tilt sensor mode because the focal length is longer than the diameter of the telescope in most cases.

Once the segments are cophased as well as cofocused, the segment alignment system will be removed. The remaining task is to maintain the phasing status of the segmented mirror. The minimum-norm least-squares method or the damped least-squares method is available for this purpose. Because of the distortions in telescope structure, the droop mode of the segmented mirror drifts slowly. These drifts correspond to position changes in focus mode. We have to employ Eq. (7) again with the aid of a segment alignment system to control it. In practice, the phase calibration procedures are complicated by the fact that sensor readings change with the elevation and the temperature.

\section{Experimental System for Segmented-Mirror Active Optics}

An experimental system for segmented-mirror active optics (shown in Fig. 3) was developed in China in $1998 .^{8}$ It is composed of three hexagonal segments with $250-\mathrm{mm}$ diagonals, which form a segmented mirror with a 3000-mm spherical curvature radius and a 500-mm-daim circumcircle. The differences of the segments' curvature radii are less than $0.025 \mathrm{~mm}$, and the figure error of each segment is about $\lambda / 40 \mathrm{rms}^{9}$ (equal to $16.25 \mathrm{~nm}$ for $\lambda=650 \mathrm{~nm}$ ). The surface errors of mirror $\mathrm{M}_{2}$ and mirror $\mathrm{M}_{3}$ are both less than $\lambda / 40 \mathrm{rms}$ at well. Therefore, the optical imaging qual-

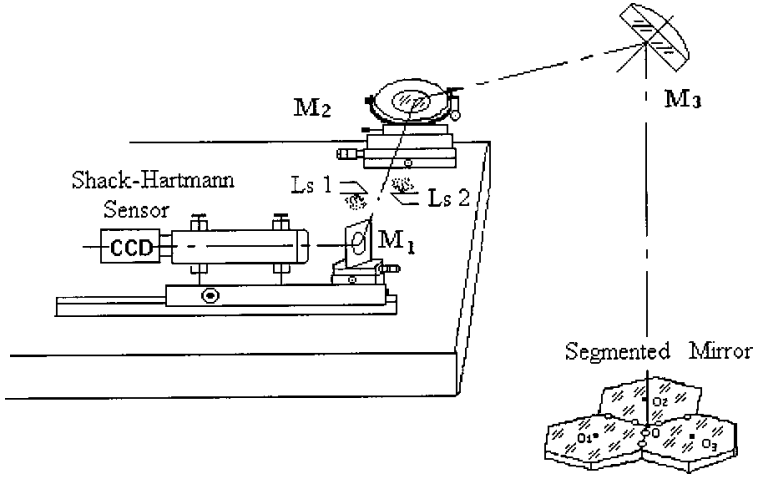

Fig. 3 The schematic layout of the experimental system for segmented mirror active optics. First, the point light source is placed at position Ls1 facing toward mirror $M_{1}$ to generate the standard Shack-Hartmann spots for the Shack-Hartmann system calibration, then the point light source is rotated along its axis to position Ls 2 facing toward mirror $\mathrm{M}_{2}$ to illuminate the segmented mirror and generate the measurement Shack-Hartmann spots for aligning the mirror segments.

ity of the experimental system will be good enough for diffraction limit, with a Strehl ratio of 0.8 , if the segmented figure is cophased within 17-nm rms.

Each segment is supported by three displacement actuators, and the relative heights between segments are measured by six capacitive sensors, which are the similar type used in Keck telescopes. The measurement accuracy of the capacitive sensor is about 3-nm rms, but its stability is very poor, because the capacitive sensors are not sealed in our system. Therefore, the sensor readings are very unstable and drift quickly with ambient turbulences. The displacement actuator is a step-motor-driven steel lever framework with flexible junctures. Its smallest controllable step size is $4.6 \mathrm{~nm}$, and its working range is about $8 \mu \mathrm{m} .{ }^{10}$ Once the primary mirror is properly phased, the diffraction-limited image can be obtained and maintained for only about 30 min with the closed loop active control system engaged.

A traditional Shack-Hartmann test is used to measure the tip/tilt of the segments and provide information for aligning (cofocusing) the primary mirrors. Since the segmented mirror is not continuous, we cannot reconstruct the wavefront (i.e., the segmented mirror surface) from discrete slope data, because it does not satisfy the Neumann Boundary problem of the Poisson equation. However, we can compute the tilts and defocuses of the segments by comparing the measurement Shack-Hartmann spots with the standard Shack-Hartmann spots. The similarities of the triangles in Fig. 2(a) between the measurement and the standard determine the defocuses of the segments, and the similarity of the triangle composed by the three-triangle geometric centers determines the tilts of the segments. The centroiding accuracy of the CCD camera is about $1 / 15$ pixel $(0.67 \mu \mathrm{m})$ $\mathrm{rms}$, and the focal length of the lenslet array is $128 \mathrm{~mm}$. The angular error of light at the exit pupil is $1.1 \mathrm{arc} \mathrm{sec}$, which corresponds to the 0.07 -arc second tip/tilt error of the segments. ${ }^{8}$ The size of the point-light source is $0.02 \mathrm{~mm}$ diam. It determines that the largest diffraction aperture for this system is $238 \mathrm{~mm}$ in diameter. In practice, we adopted an aperture of 220-mm diameter. 


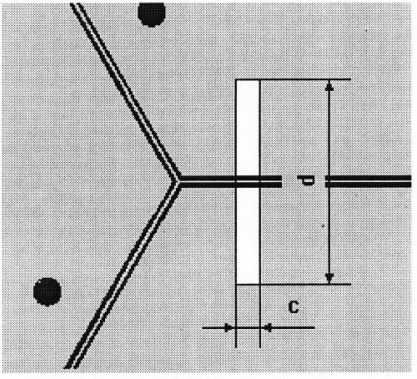

(A)

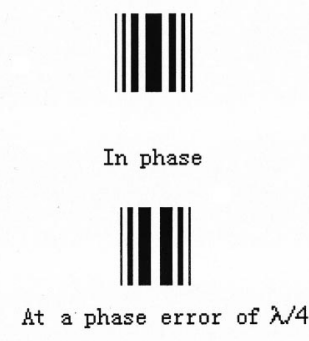

[B]

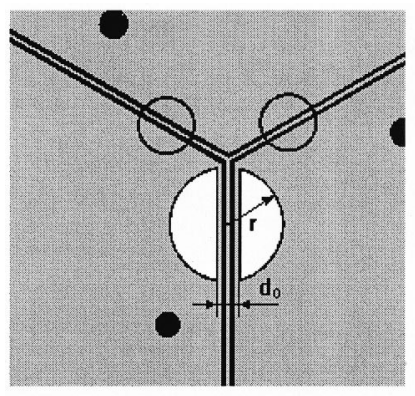

[A]

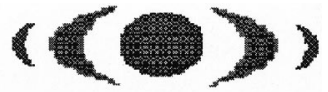

In phase

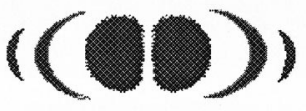

[B]
At a phase error of $\lambda / 4$

Fig. 4 Phase calibration employing the single-slit method. The segmented mirror is covered by an opaque paper with a $c \times d$ exposing slit.

\section{Phase Measurements in Phase Calibration}

In this section, we discuss how to obtain the sensor reading in phase at each sensor site. The phase measurement methods can be classified into two types: absolute measurement and relative measurement. On the other hand, the phase measurements can also be categorized as noncontact measurement and contact measurement. Optical measurements typically fall into the first category and the mechanical measurements into the second one. Now we introduce the phase measurement methods we developed, which can be used for SSPC.

\subsection{Optical Means}

\subsubsection{Single-slit method}

A single slit is placed over the intersegment mirror surface at a calibration site as shown in Fig. 4(a). If the two segments are in phase on this local site, we then obtain the standard single-slit diffraction fringe pattern as shown in Fig. 4(b). If they are not in phase, we obtain the usual double-slit interference fringe pattern. This method suffers from the halfwave ambiguity problem for the monochromatic light source, so we can only determine the edge height error to within an integer number of halfwaves. Thus, this method is only suitable for fine sensor phasing calibration when the edge height errors have already been reduced to less than half of a wavelength. This method belongs to relative measurement. We adjust the actuators to make the segments in phase at the local calibration area, and then we record the sensor reading accordingly once the cophasing pattern shown in Fig. 4(b) appears. This simple but efficient method can reach very high measurement accuracy. Judging by human eye, the sensor readings in phase can be determined within 10-nm rms. In addition, this method has a very low requirement for segment alignment. It works as long as the star images from the two individual segments are roughly superimposed within a small defocusing distance from the focal plane, although a very good alignment is more helpful. The breadth of the fringes is closely related to parameter $c$ and parameter $d$; the larger the ratio $d / c$ is, the sharper the fringes are. Considering the integral effect of the interference pattern, we usually adopt a large value of ratio $d / c(>12)$ and a long slit when the segment alignment is poor. Nevertheless, in fine calibration when the segment alignment is improved, we adopt a smaller value of ratio $d / c$ and a shorter slit so that the
Fig. 5 Phase calibration employing the circular-aperture method. The segmented mirror is covered by an opaque paper with a circular exposing aperture that straddles the adjacent edges.

fringe pattern is produced by a small area near the sensor location to fulfill the idea of SSPC strategy. In our experiment system, we select: $d=120 \mathrm{~mm}$ and $c=9 \mathrm{~mm}$ in coarse calibration, and $d=50 \mathrm{~mm}$ and $c=6 \mathrm{~mm}$ in fine calibration.

\subsubsection{Circular-aperture method}

As shown in Fig. 5(a), we place a mask with circular clear apertures over the mirror surface, such that each aperture straddles the adjacent edges of two segments at each sensor location. If the two halves of the area covered by an aperture are in phase, we obtain the standard Airy diffraction pattern of single circular aperture with a cross-band shown in Fig. 5(b); otherwise, we obtain a two-aperture interference pattern. Like the single-slit method, this method is suitable for fine phasing calibration. It also has a very low requirement for segment alignment and has the halfwave ambiguity problem for a monochromatic light source. This method uses a relative measurement, and its precision is equivalent to that of the single-slit method. In addition, the interference pattern is closely related with parameters $r$ and $d_{0}$. In our experiment system, $r=25 \mathrm{~mm}$ and $d_{0}=10 \mathrm{~mm}$.

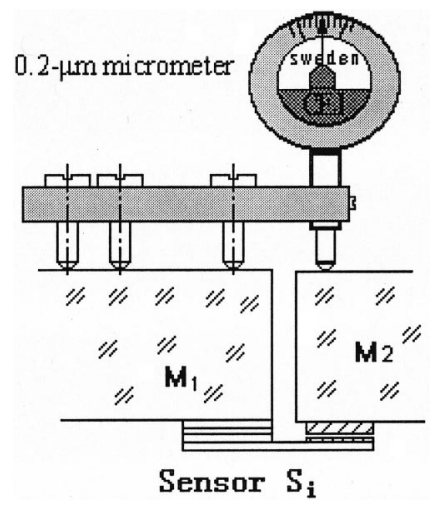

Fig. 6 Phase calibration employing a spherometer with a $0.2-\mu \mathrm{m}$ micrometer. To calibrate sensor $S_{i}$, the spherometer is first placed on $M_{1}$ to determine the zero point of micrometer $S_{0}$. Then the spherometer is put onto the site right over sensor $S_{i}$, straddling two segments as shown in this figure. By adjusting the actuators to make the pointer of the micrometer reading remains on $S_{0}$, the reading of sensor $S_{i}$ is recorded as the desired sensor readings. 


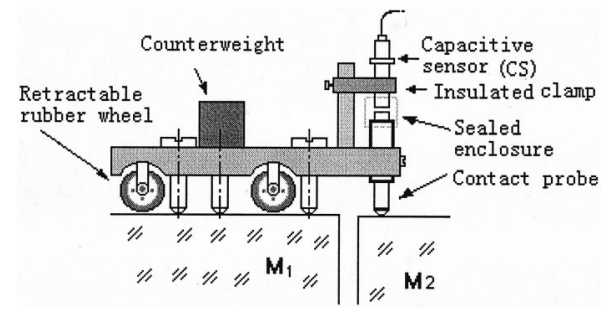

Fig. 7 A schematic drawing of the phase calibration carriage for the phase calibration of a large segmented mirror. This figure is similar to Fig. 6 except that the $0.2-\mu \mathrm{m}$ micrometer is replaced by the phase calibration carriage. The phase calibration carriage is first put on a segment, such as $\mathrm{M}_{1}$, to determine the zero point of CS, $S_{\alpha}$. Then the carriage moves onto the calibration site over sensor $S_{i}$ (the $i^{\prime}$ th sensor). The reading of CS is recorded as $S_{\beta}$, and the reading of the $i^{\prime}$ th sensor on the back of the segment is recorded as $S_{i}$. Thus, the desired sensor reading of this point is $S_{i}+S_{\beta}-S_{\alpha}$. Rubber wheels are retracted off the mirror while in calibration, and stick out to lift the capacitive spherometer off the mirror surface after each calibration, moving from one site to another.

\subsection{Mechanical Means}

A spherometer-type tool can be used as a mechanical means in phase calibration, but a common spherometer, with $0.01-\mathrm{mm}$ precision, is not precise enough for phase measurement. In our experimental system, we use a spherometer with a $0.2-\mu \mathrm{m}$ precision micrometer, ${ }^{11}$ as shown in Fig. 6 , which enables us to make a relative calibration of the sensor readings in phase within $30 \mathrm{~nm}(15-\mathrm{nm} \mathrm{rms})$. This relative measurement is free of the halfwave ambiguity.

To apply this method to the phase calibration of a large segmented telescope, we have designed a device shown in Fig. 7. The mirror in Fig. 7 is set to point to zenith; a rubber-wheel carriage (or a similar device) moves from one calibration site to another automatically. First, we put the phase calibration carriage on a single mirror segment to calibrate the zero point of a capacitive sensor (CS) shown in Fig. 7, $S_{\alpha}$, and then the carriage moves onto the calibration site over sensor $S_{i}$ bridging two segments, which is not shown in Fig. 7. Meanwhile, the reading of CS is recorded as $S_{\beta}$, and the reading of sensor $S_{i}$ on the back of the segment is recorded as $S_{i}$. Thus, the desired sensor reading of this site is

$S_{i}^{\text {in phase }}=S_{i}+S_{\beta}-S_{\alpha}$.

In practice, the scale coefficients of the sensor readings between the sensor CS and the telescope sensors should be considered. Thus, if the sensor reading of this site is $S_{i}^{\text {in phase }}$, the local intersegment area at this site will be in phase. Since the precision of the capacitive sensor is only about several nanometers, we can expect that this absolute phase calibration method will have a very good accuracy. However, this method is a contact measurement, so it requires the mirror surface to be clean enough, and the material of the contact probe should be selected to avoid damaging the mirror surface but still have a very good rigidity. Therefore, this method needs further study in the case of the large segmented mirror telescopes.

\section{Advantages of the SSPC Method in Phase Calibration}

The SSPC method is free of the influence of the residual tilt errors of the segment alignment. Of course, too large segment misalignments will also affect the precision of SSPC, but this effect is reduced in importance because of the iteration process in phase calibration.

The accuracy of SSPC has little to do with the segment figures. A sensor reading in phase only depends on the phasing of the local areas of the two segments that are close to the sensor; it does not depend strongly on the other areas of the two segments. Very large segment aberrations will, of course, affect the cophasing of the segment, but this is not a problem unique to the SSPC method. There exists an optimum piston mode for each segment surface, which best fits the cophasing surface of the whole mirror. We notice that there are twelve sampling points at the six edges of an inside segment. That will make the segment surface conform well to the optimum-phasing surface in the leastsquares sense.

Normally, the precision of calibration should be equivalent to or higher than the precision of maintenance in an active control system. For a mirror scheme shown in Fig. 1, the SSPC employs 168 phase measurements to achieve the phase calibration, so that its precision just competes with that of the telescope active control system.

Besides relative measurement, the SSPC does not exclude to absolute measurements. Besides the method shown in Fig. 7, we can employ the optical calibration method used in Keck telescopes ${ }^{2}$ to measure the edge phase errors at each calibration point shown in Fig. 1; then we calculate the desired sensor readings referring to Eq. (9).

\section{Phase Calibrations in Our Experimental System}

In this section, we demonstrate the validity of the SSPC method and its cophasing algorithm. To avoid the halfwave ambiguity, the mechanical sensor, a $0.2-\mu \mathrm{m}$ spherometer, is used in the coarse phase calibration before switching to fine phase calibration of the circular-aperture method or singleslit method. The calibration site map of the circularaperture method is shown in Fig. 2.

Because of the poor stability of the capacitive sensors used, the phase calibration in our experiment has its own feature: the time for phase calibration should be as short as possible to avoid excessive sensor reading drifts. The SSPC method does not require the segmented mirror to be precisely in phase for obtaining the desired sensor readings, but we still need to calibrate all the sensors one by one. This is a time-consuming procedure for manual calibration with a simple tool. To reduce the time for calibration, the number of the sensors we actually calibrate should be reduced to as few as possible. In practice, only two of the desired sensor readings (sensor 3 and sensor 5, for example) are manually calibrated, the others can be obtained by calculations from the alignment sensor readings $\mathbf{S}_{\text {align }}$ and the two desired readings we have obtained. Theoretically, the differences between the cofocus sensor readings and the cophase sensor readings are a set of parallel piston errors. 


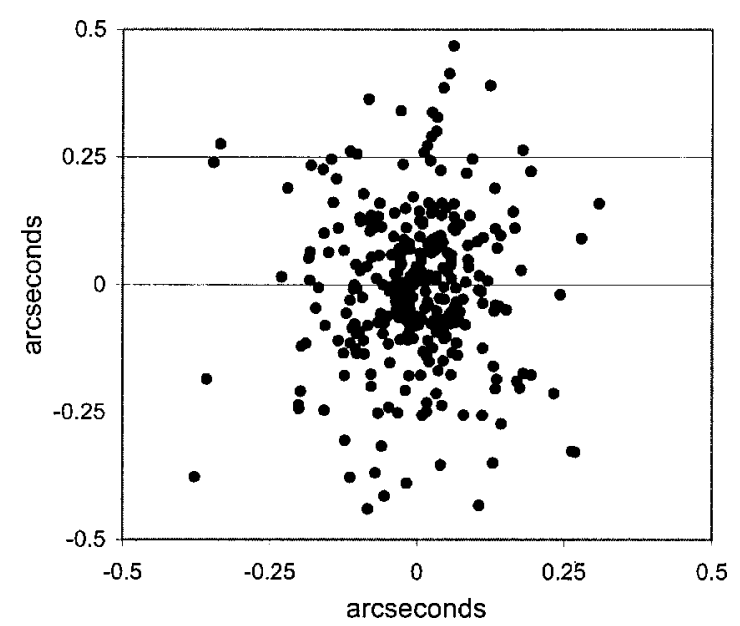

Fig. 8 The spot diagram of the segmented primary mirror of the experimental system generated by the residual tip/tilt errors of the segment alignment system. Atmospheric seeing influence is not removed in this diagram. This shows that this active alignment system can maintain the image of the segmented mirror within a disk of 0.216 arc sec rms radius.

Tricks are needed to obtain $\mathbf{S}_{\text {align }}$. Because of air turbulence, the CCD measuring error, and the actuator position errors, it is difficult to obtain a desirable alignment. Although the temporal integration algorithm was adopted to reduce the seeing effects, the rms image radius of the segment alignment of our system is still about 0.216 arc sec (shown in Fig. 8). A 0.216 arc sec tilt error corresponds to a $57-\mathrm{nm}$ sensor reading difference on an edge, which is obviously not good enough to calculate the desired sensor readings. To improve this, a Shack-Hartmann grid shown in Fig. 2 is employed to improve the measuring accuracy of the CCD camera; and a threshold of $0.04 \mathrm{arc} \mathrm{sec}$ is set for alignment selection. The sensor readings are selected as the desirable alignment sensor readings $\mathbf{S}_{\text {align }}$ only when the tilts of the three segments are all within the $0.04 \mathrm{arcsec}$ to the fiducial axis. A 0.04 arc sec tilt error corresponds to a $10-\mathrm{nm}$ sensor reading difference on an edge. This method is no longer efficient for the system with a large number of segments, because the probability to obtain the desirable alignment for all the segments tends to zero when the number of mirror segments increases. However, we do not plan to apply this trick in the phasing calibration of a large segmented telescope mirror, because according to the SSPC method the phased sensor readings $\mathbf{S}_{\text {in phase }}$ for segment phasing do not depend on segment alignment $\mathbf{S}_{\text {align }}$. This trick is employed because we want to use $\mathbf{S}_{\text {align }}$ to calculate the sensor readings in phase from only two sites of calibration so that we can speed up the calibration process.

The following procedures are designed for performing an efficient phase calibration. First, keep the alignment system running continuously to align the three segments until a desirable alignment is selected, then the six sensor readings are recorded in the following format

$\mathbf{S}_{\text {align }}=\left(\mathbf{s}_{f 1}, \mathbf{s}_{f 2}, \mathbf{s}_{f 3}, \mathbf{s}_{f 4}, \mathbf{s}_{f 5}, \mathbf{s}_{f 6}\right)$.

These sensor readings represent the positions of the segments with a desirable alignment, which are selected in

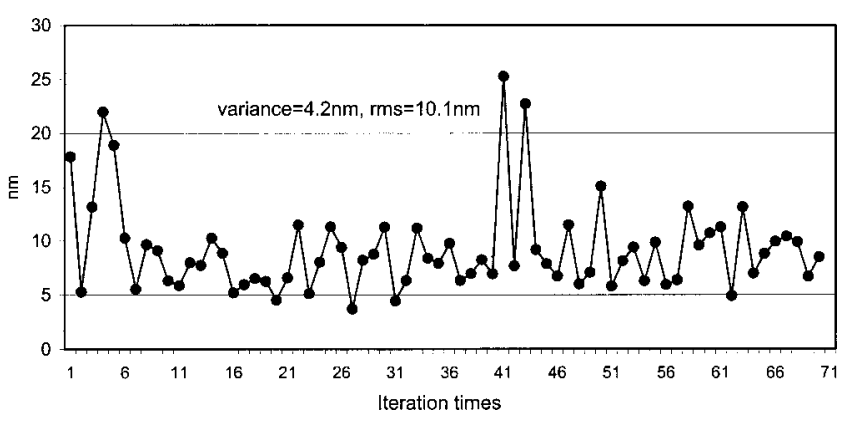

Fig. 9 The residual $\mathrm{rms}$ segmented surface errors obtained from the active control system of the experimental system for segmented mirror active optics in China. The time interval of iteration is about $20 \mathrm{sec}$. The rms value of all the rms surface errors is $10 \mathrm{~nm}$.

real-time automatically according to the 0.04-arc sec threshold. Next, serially calibrate (as the SSPC method permits) two of the three innermost sensors, sensor 5 and sensor 3 shown in Fig. 2, for example, and obtain the desired sensor readings $s_{05}$ and $s_{03}$. Mathematically, there are only "parallel piston distances" between the segment positions in phase and the segment positions in alignment. Therefore, the sensor reading in phase of sensor 1 is

$\mathbf{s}_{01}=\mathbf{s}_{f 1}+\left(\mathbf{s}_{05}-\mathbf{s}_{f 5}\right) \mathbf{k}_{15}$.

The sensor reading in phase of sensor 4 is

$\mathbf{s}_{04}=\mathbf{s}_{f 4}+\left(\mathbf{s}_{03}-\mathbf{s}_{f 3}\right) / \mathbf{k}_{34}$.

The sensor reading in phase of sensor 2 is

$\mathbf{s}_{02}=\mathbf{s}_{f 2}+\left(\mathbf{s}_{05}-\mathbf{s}_{f 5}\right) \mathbf{k}_{25}-\left(\mathbf{s}_{03}-\mathbf{s}_{f 3}\right) \mathbf{k}_{23}$.

The sensor reading in phase of sensor 6 is

$\mathbf{s}_{06}=\mathbf{s}_{f 6}+\left[\left(\mathbf{s}_{05}-\mathbf{s}_{f 5}\right) \mathbf{k}_{25}-\left(\mathbf{s}_{03}-\mathbf{s}_{f 3}\right) \mathbf{k}_{23}\right] / \mathbf{k}_{26}$,

where $\mathbf{k}_{15}=1.090866, \mathbf{k}_{26}=0.91940, \mathbf{k}_{34}=0.927264, \mathbf{k}_{25}$ $=1.072208$, and $\mathbf{k}_{23}=0.997894$. These scale coefficients between sensors are obtained by experimental calibrations. Therefore, the sensor readings in phase for our experiment system are

$\mathbf{S}_{0}=\left(\mathbf{s}_{01}, \mathbf{s}_{02}, \mathbf{s}_{03}, \mathbf{s}_{04}, \mathbf{s}_{05}, \mathbf{s}_{06}\right)$.

$\mathbf{S}_{0}$ are the desired readings that should be included in the control equation developed in Sec. 2.2. The next step is to run the active control system to maintain the sensor readings to the desired sensor readings.

According to the deviations of sensor readings from the desired sensor readings, we can calculate the position errors of the actuators from Eq. (1). The rms surface error of the segmented mirror introduced from an active optics control system can then be expressed by ${ }^{7}$

$$
\begin{aligned}
\sigma_{\text {surface }}^{2}= & \frac{1}{54} \sum_{i=0}^{2}\left[7\left(p_{3 i}^{2}+p_{3 i+1}^{2}+p_{3 i+2}^{2}\right)-\left(p_{3 i} p_{3 i+1}\right.\right. \\
& \left.\left.+p_{3 i+1} p_{3 i+2}+p_{3 i+2} p_{3 i}\right)\right] .
\end{aligned}
$$


The phase calibration is one of the key techniques in our experimental system. It takes only about 5 min to perform a phase calibration, and another one or two minutes to phase the segments. The precision of phase calibration is about $15-\mathrm{nm} \mathrm{rms}$, and the rms surface error mainly from the active control system is only about $10 \mathrm{~nm}$ as shown in Fig. 9. A clear diffraction-limited pattern was easily obtained on a 220-mm-diam aperture over the segmented mirror with a 20- $\mu \mathrm{m}$-diam, 650-nm-wavelength light source.

\section{Discussions}

The sensor-by-sensor phase calibration method is a new conceptual phase calibration method proposed. The SSPC method is free of the influences of residual tip/tilt errors, has little to do with the segment figure, and provides a competent precision for an active control system. The biggest challenge for the segmented mirror on a real star is that the centroiding accuracy of the Shack-Hartmann spots is affected by the air turbulence. However, in SSPC, we do not depend on the segment alignment to calibrate the sensor readings in phase, and the segment alignment only takes a small weight in the segment phasing control according to Eq. (7). In other words, the segment alignment is not a main process in the segment phasing control, so the air turbulence effect to the centroiding accuracy of ShackHartmann spots is not very important in SSPC. The experimental system for segmented mirror active optics has demonstrated the validity of this SSPC method. It takes only about 5 min to complete the phase calibration and about 2 min to phase the segments.

However, some issues need further study. The spot diagram in Fig. 8 is still somewhat dispersive and irregular. The dispersion of the spot diagram is contributed by the air seeing, which affects the CCD centroiding of ShackHartmann spots, and the environmental turbulence, which affects the sensors. It is also caused by the release of the resistant torque stress when the actuator motor begins to inversely rotate in active control process. The resistant torque stress differs considerably in different actuators, and that makes the shape of the spot diagram irregular. For our case, it is elongated in one direction. Further research on qualitatively comparing the stability of the diffraction limit status for our system is needed if the environmental influence to the capacitive sensors is highly reduced.

\section{Acknowledgments}

This work is undertaken with the auspices of the program "Research on technology of active optics in large telescopes," funded by the National Science Foundation of China, authorization number 19333030, Math-Physical Department of the Chinese Academy of Sciences. The author

is indebted to many people. The author cordially thanks Professor Dingqiang Su for his advice and support to this work. The experimental system was performed with the assistance of Professor Zhenchao Zhang, Professor Yuangen $\mathrm{Qu}$, and senior engineer Shuying Yang of Nanjing Institute of Astronomical Optics and Technology (NIAOT), the Chinese Academy of Sciences. The author especially thanks Professor Glenn Boreman and Professor Jannick Rolland at CREOL/School of Optics, University of Central Florida, and Professor Shri Kulkarni, Dr. Christopher Koresko, and Dr. Josh Chen at the California Institute of Technology for their kind help and support. The author cordially thanks all the reviewers of this work for their comments and advice.

\section{References}

1. R. H. Minor, A. A. Arthur, G. Gabor, H. G. Jackson, R. C. Jared, T. S Mast, and B. A. Schaefer, "Displacement sensors for the primary mirror of the W. M. Keck telescope," Proc. SPIE 1236, 1009-1017 (1990).

2. G. Chanan, T. Mast, J. Nelson, R. Cohen, and P. Wizinowich, "Phasing the mirror segments of the W. M. Keck Telescope," Proc. SPIE 2199, 622-637 (1994)

3. G. Chanan, M. Troy, F. Dekens, S. Michaels, J. Nelson, T. Mast, and D. Kirkman, "Phasing the mirror segments of the Keck telescopes: the broadband phasing algorithm," Appl. Opt. 37(1), 140-155 (1998).

4. G. Chanan, M. Troy, and E. Sirko, "Phasing discontinuity sensing: a method for phasing segmented mirrors in the infrared," Appl. Opt. 38(4), 704-713 (1999).

5. T. S. Mast and J. E. Nelson, "Figure control for a fully segmented telescope mirror," Appl. Opt. 21(14), 2631-2641 (1982).

6. W. Zou, "Generalized figure-control algorithm for large segmented telescope mirrors," J. Opt. Soc. Am. A 18(3), 638-649 (2001).

7. W. Zou, "Figure control for large segmented telescope mirrors," Master's thesis, Chinese Academy of Sciences, Nanjing Astronomical Instruments Research Center, Nanjing (1996).

8. D. Su, W. Zou, Y. Qu, Z. Zhang, L. Wang, S. Yang, and Y. Yao, "Experimental system of segmented-mirror active optics," Proc. SPIE 4003, 417-425 (2000).

9. L. Wang and Y. Rao, "Fabrication and testing of the optical system of the experimental system for segmented mirror active optics," technique report, Shanghai Observation, Chinese Academy of Sciences, Shanhai, China (1998)

10. Y. Qu, "Design of nanometer level displacement actuator and its precision analysis," technique report, Nanjing Astronomical Instruments Research Center, Chinese Academy of Sciences, Nanjing, China (1998).

11. MIKROKATOR 500 A-7, Sweden.

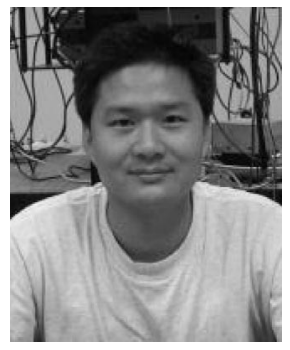

Central Florida
Weiyao Zou received his BS in optical instruments from Tianjin University and his MS in astrophysics from the Chinese Academy of Sciences. His research fields include active and adaptive optics, optical testing, and large astronomical instrumentation and numerical method in optics. Currently he is a graduate student working on the technique of local curvature measurements and shape extraction in the School of Optics/CREOL, University of 